\title{
MIP and CT Scanning Characterization of Pore Structure of Tight Volcanic Rocks
}

\author{
Yunliang YU a, Haibo XU ${ }^{\text {a,1 }}$, Ye BAI ${ }^{\text {b }}$, Huijing ZHANG $^{\text {c }}$, Wenqing NIU ${ }^{\text {a }}$ and Liya \\ TIAN $^{\text {a }}$ \\ ${ }^{a}$ College of Earth Sciences, Jilin University, Changchun, China \\ b Jilin Coal Geological Geophysical Prospecting Measure Team, Changchun, China \\ c College of Chemistry, Jilin University, Changchun, China
}

\begin{abstract}
Volcanic rocks are unconventional oil and gas reservoirs, and the pore structure of the rock is relatively complex. In this study, we selected samples from CS105 well and DS9 well from Yingcheng Formation volcanic reservoirs in the southern Songliao Basin to study the pore structure. First, we performed experiments on two rock samples using computed tomography (CT), and then measured the porosity and density of the two rock samples using mercury intrusion porosimetry (MIP). According to the data obtained by micron computed tomography (Micro-CT), Avizo 2019.1 software was used to perform three-dimensional (3D) reconstruction on the micron level of the two wellhead samples. Analyze the microscopic pore structure of the rock sample and compare it with the porosity of the sample obtained by the mercury injection method. Compared with MIP, Micro-CT more accurately characterizes the pore structure and porosity of volcanic rocks.
\end{abstract}

Keywords. Volcanic rock, mip, micro-ct, pore structure

\section{Introduction}

In recent years, more and more people have paid attention to unconventional oil and gas reservoirs [1]. As unconventional oil and gas reservoirs, tight volcanic rocks have received more and more attention [2]. Because volcanic rock is a low-permeability rock, its rock structure is more complicated [3]. The pore structure has a great influence on the reservoir and migration of oil and gas. Due to the limited pressure, the pores of the rock samples measured by MIP are mostly large and medium pores [4], and only connected pores can be tested, and the obtained porosity value is low [5]. CT is a method developed in recent years to use X-rays to quickly and non-destructively scan a rock sample in an all-round, large-scale range, and finally use the numerical value of the scanned image to reconstruct the $3 \mathrm{D}$ pore structure characteristics [6]. This technology can perform micro-CT analysis for samples of different sizes, obtain micron and millimeter-level multi-scale pore structure features, and accurately locate the exact positions of different pores in the sample [7]. To avoid the disadvantage that MIP cannot reflect the characteristics of micro-pore structure heterogeneity in tight reservoirs [8].

\footnotetext{
${ }^{1}$ Haibo Xu, Corresponding author, College of Earth Sciences, Jilin University, Changchun 130061, China; E-mail: xuhb19@mails.jlu.edu.cn.
} 
The Songliao Basin is located in the northeast of China (figure 1A). The dacite sample of CS105 well and andesite sample of DS9 well are respectively from Changling and Dehui fault depression of Yingcheng Formation in the southern Songliao Basin (figure 1B). In this study, CS105 and DS9 samples were studied (figure 1C). According to the data obtained by Micro-CT, the Avizo 2019.1 software was used to reconstruct and display the 3D samples of the two wellheads. Use the Volume Rendering module in the software to qualitatively display the 3D spatial distribution characteristics of the pores. The Label Analysis module in the software was used to quantitatively analyze the micro pore structure characteristics of rock samples, and the sample porosity obtained by MIP was compared and analyzed. It explains the low porosity of the rock samples obtained by MIP, which is of great significance for understanding the pore structure of tight volcanic rocks and the ability of oil and gas storage and migration.
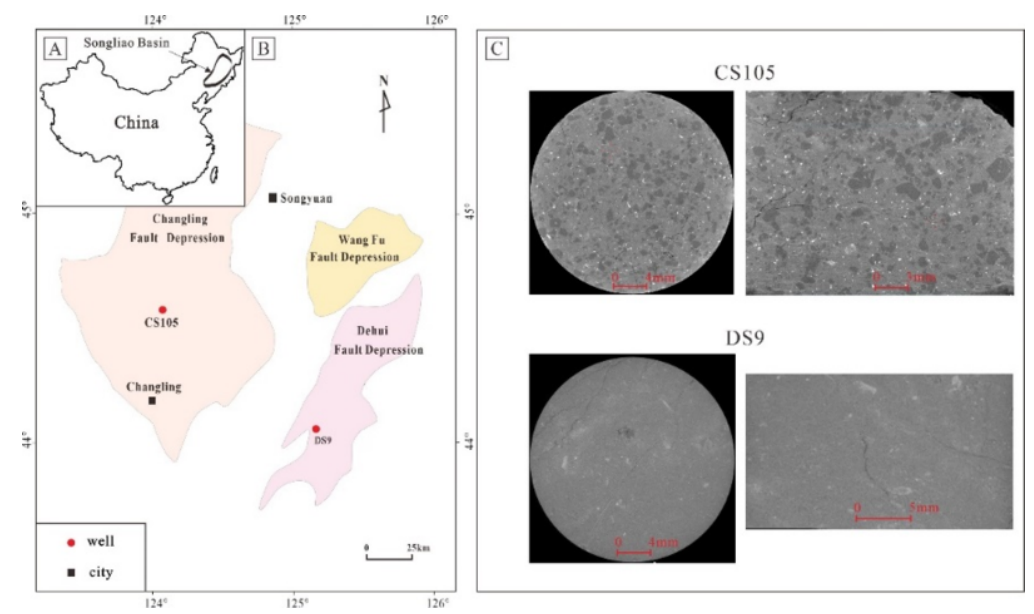

Figure 1. Location and sample images of the CS105 well and DS9 well in the Songliao Basin. (A) Location of the Songliao Basin in China. (B) Location of the CS105 well and DS9 well in the Songliao Basin. (C) Images of volcanic rock samples from the CS105 well and DS9 well.

\section{Materials and Methods}

\subsection{Samples}

We selected the dacite samples of the CS105 well and andesite samples of the DS9 well from the volcanic reservoirs of Yingcheng Formation in the southern Songliao Basin, which are both extrusive rocks. First, we conducted thin-section analysis on two rock samples to obtain the physical properties of the reservoir rocks. Then we used MIP and Micro-CT to analyze the pore structure characteristics of the two samples.

\subsection{Casting flakes and MIP}

The cast sections were identified using a Zeiss polarizing microscope, and the porosity and density of the rock samples were measured using a model 9310 automatic mercury injection apparatus. The working pressure of the 9310 automatic mercury injection 
instrument is $0.0035-206.843 \mathrm{MPa}$, the resolution is $0.1 \mathrm{~mm}^{3}$, and can measure the pore size of $7.2 \mathrm{~nm}$ or more [9].

\subsection{Micro-CT}

X-ray CT scanning technology uses X-rays to scan and image an object and reconstruct different slices with the help of computer technology. This technology can perform non-destructive testing of the composition and structure of non-transparent materials, and is also known as X-ray computer tomography technology [10].

The experimental instrument for CT scanning uses the micron CT scanner phoenix v|tome|xm from Shanghai Yinghua Testing Co., Ltd., China. The maximum scanning sample diameter of the micron CT instrument is $500 \times 600 \mathrm{~mm}^{2}$, and the maximum 3D scanning field of view is $420 \times 400 \mathrm{~mm}^{2}$. The rock sample in this test micron CT scan experiment is a cylinder with a diameter of $2.5 \mathrm{~cm}$, and the resolution is $13 \mu \mathrm{m}$.

\section{Results and Discussions}

\subsection{Physical Characteristics of Reservoir Rocks}

Through the identification of rock slices, we have observed that the CS105 sample is dacite with a porphyritic structure and a massive structure. The phenocrysts account for $25 \%$ of the total mineral content, and the plagioclase part of the phenocrysts has sericite alteration, the grain size is about $1 \mathrm{~mm}$, and the maximum can reach $3 \mathrm{~mm}$. The quartz is subangular to subcircular with a particle size of about $1 \mathrm{~mm}$ (figure $2 \mathrm{~A}$ ). The DS9 sample is andesite with porphyry structure and an andesite matrix. Phenocrysts account for $15 \%$ of the total mineral, plagioclase is angular, the grain size of about $0.5-2 \mathrm{~mm}$. The quartz is subangular to subcircular, with a particle size of about 1-2 $\mathrm{mm}$ (figure $2 \mathrm{~B}$ ).
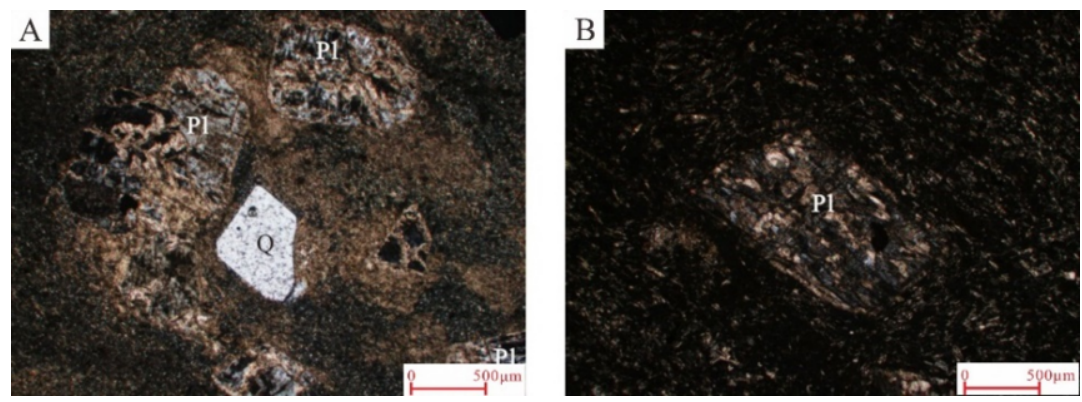

Figure 2. Thin sections of rock. (A) CS105 sample. (B) DS9 sample.

The porosity and density of the CS105 sample measured by MIP were $2.3 \%$ and $2.64 \mathrm{~g} / \mathrm{cm}^{3}$, and the porosity and density of the DS9 sample were $3.3 \%$ and $2.53 \mathrm{~g} / \mathrm{cm}^{3}$, respectively. Mercury can enter the pores and throats of the rock when the pressure reaches the entry pressure of the connected pores and throats. In actual measurement, due to certain restrictions on pressure, the tested rock pores are often dominated by large and medium pores, and independent pores (not connected to other pores through 
throats) cannot be measured. Therefore, the measured porosity of the rock sample is much smaller than the actual value, which cannot accurately characterize the pore structure of the tight volcanic rock. Volcanic rocks have many micron-level pore throats. Therefore, we use Micro-CT to accurately characterize the pore structure of tight volcanic rocks.

\subsection{Micro-CT Pore Structure Characteristics}

Volcanic rocks have strong heterogeneity, most of the pores and throats are in the micron level, and locally developed in the millimeter level [11]. Many pores have irregular geometric shapes and uneven spatial distribution, which affects the physical properties of volcanic rocks [12]. In order to better understand the pore structure characteristics of tight volcanic rocks, we used Micro-CT to analyze the CS105 sample and DS9 sample. After obtaining the pore structure information of the sample through the Micro-CT equipment, the VGStudio MAX 3.3 software is used to process the 3D pore structure characteristics of the rock sample. Then select a partial area of the sample to generate a data file, and then import this data file into the Avizo 2019.1 software. The Volume Rendering module of Avizo 2019.1 software was used to obtain the $3 \mathrm{D}$ reconstruction map and the $3 \mathrm{D}$ pore distribution map of the rock sample, and the Label Analysis module was used to analyze the pore structure characteristics of the rock sample [13].

Figure 3 shows the 3D reconstruction image of the CS105 sample and the DS9 sample. The $3 \mathrm{D}$ reconstruction volume of CS105 sample is $11.7 \times 11.7 \times 10.4 \mu^{3}$, and the $3 \mathrm{D}$ reconstruction volume of DS9 sample is $10.467 \times 10.467 \times 10.467 \mu^{3}$. In the $3 \mathrm{D}$ reconstruction model, black represents pores and cracks, gray represents matrix, and white represents minerals (figure 3). We can observe that the two samples develop more pores and cracks, and the pores and cracks of the CS105 sample are better than those of the DS9 sample.
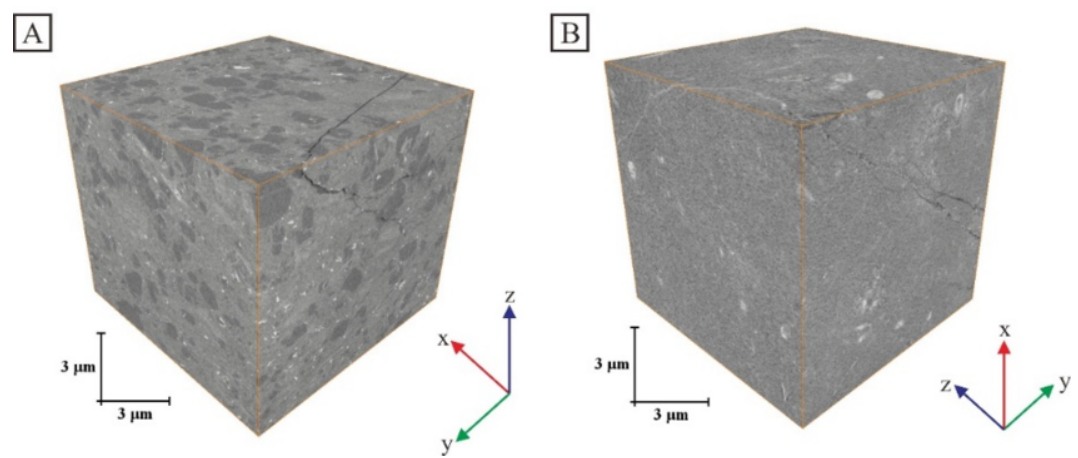

Figure 3. 3D reconstruction of rock samples by Micro-CT. (A) CS105 sample. (B) DS9 sample.

Figure 4 shows the spatial distribution characteristics of the pores of the CS105 and DS9 samples. We can observe that the CS105 sample has more pores of medium level and above, and the pores are well developed (figure 4A). The DS9 sample has more pores below medium grade and poor pore development (figure 4B). According to the results of quantitative analysis (table 1), both CS105 and DS9 samples developed more micron-level pores. The number of pores, total volume of pores and total surface 
area of pores in CS105 sample were larger than those of DS9 sample. It shows that the pore development of CS105 sample is better than that of DS9 sample. The porosities of the CS105 sample and the DS9 sample obtained by Micro-CT are $15.45 \%$ and $8.49 \%$, respectively, and the porosity is larger than the porosity measured by the MIP method.
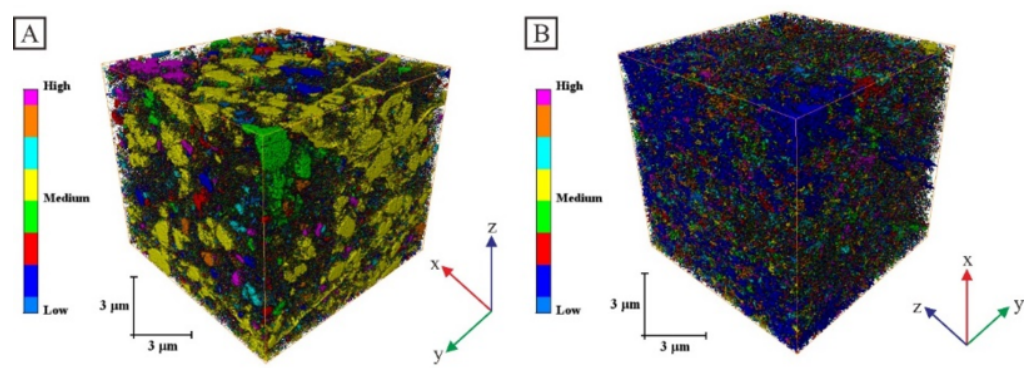

Figure 4. Pore distribution of rock samples obtained by Micro-CT. (A) CS105 sample. (B) DS9 sample.

Table 1. Pore-fracture structure characteristics of rock samples.

\begin{tabular}{llllll}
\hline CT type & Sample & Depth $(\mathrm{m})$ & Counts & $\sum$ Volume $\left(\mu \mathrm{m}^{3}\right)$ & $\sum$ Surface $\left(\mu \mathrm{m}^{2}\right)$ \\
\hline Micro-CT & CS105 & 3926.57 & 1048575 & 219.9 & 17027.4 \\
& DS9 & 2010.60 & 601826 & 97.7 & 10075.2 \\
\hline
\end{tabular}

Figure 5 shows the spatial distribution of isolated pores in CS105 sample and DS9 sample. We can observe that both the CS105 sample (figure 5A) and the DS9 sample (figure 5B) have a large number of isolated pores. According to the results of quantitative analysis, the total volume of isolated pores in the CS105 sample is $45.43 \mu \mathrm{m}^{3}$, and the total volume of isolated pores accounts for $3.19 \%$ of the total volume of the sample. The total volume of isolated pores in the DS9 sample is $69.1 \mu \mathrm{m}^{3}$, and the total volume of isolated pores accounts for $6.00 \%$ of the total volume of the sample. It shows that the isolated pores of CS105 and DS9 samples have a greater impact on the porosity of the samples. Compared with the CS105 sample, the isolated pores of the DS9 sample are more developed and have a greater impact on the porosity of the sample. Compared with the MIP method, Micro-CT measures the porosity of the sample more accurately.
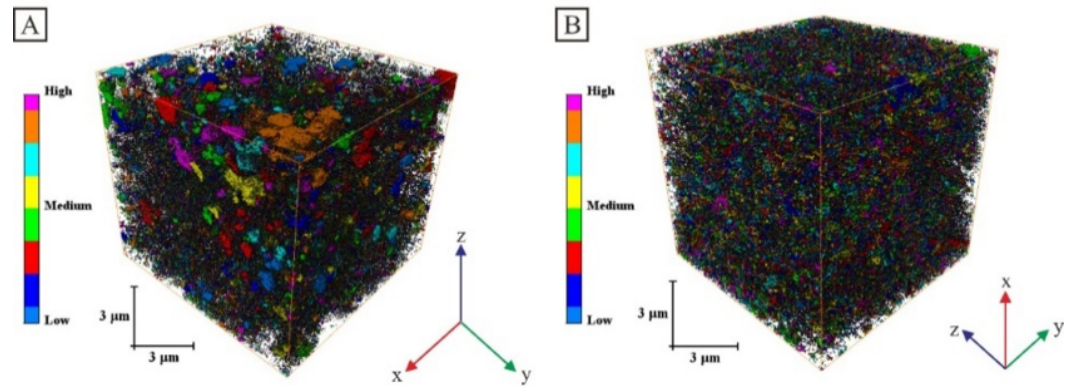

Figure 5. Isolated pore distribution of rock samples obtained by Micro-CT. (A) CS105 sample. (B) DS9 sample. 


\section{Conclusions}

The rock pore structure data obtained by Micro-CT and the 3D display map obtained by Avizo 2019.1 software show that a large number of micron-level pores are developed in volcanic rock reservoirs, which can become good oil and gas reservoirs.

Compared with Micro-CT, the range of pore sizes that can be tested by MIP is limited and independent pores cannot be measured, while rock samples usually contain a large number of isolated pores. Therefore, the measured porosity value is smaller than the actual value, and cannot fully characterize the micron-level pore structure characteristics of tight volcanic rocks.

\section{Competing interests}

The authors declare that there is no conflict of interests, we do not have any possible conflicts of interest.

\section{References}

[1] Zhang B, Shan B, Zhao Y, et al. Review of formation and gas characteristics in shale gas reservoirs. Energies. 2020 Oct; 13(20): 5427.

[2] Guo T, Huang B, Zhou W, et al. Gas-water two-phase productivity model for fractured horizontal wells in volcanic gas reservoirs with natural fractures. Energy Sources, Part A: Recovery, Utilization, and Environmental Effects. 2021 Apr; 1-19.

[3] Cid HE, Carrasco-Núnez G, Manea VC, et al. The role of microporosity on the permeability of volcanic-hosted geothermal reservoirs: A case study from Los Humeros, Mexico. Geothermics. 2021 Feb; 90(2021): 102020.

[4] Zhang Z, Kruschwitz S, Weller A, et al. Enhanced pore space analysis by use of $\mu-C T$, MIP, NMR, and SIP. Solid Earth. 2018 Nov; 9(6):1225-1238.

[5] Xin F, Xu H, Tang D, et al. Problems in pore property testing of lignite: Analysis and correction. International Journal of Coal Geology. 2021 Jul; 103829.

[6] Song R, Wang Y, Sun S, et al. Characterization and microfabrication of natural porous rocks: from micro-CT imaging and digital rock modelling to micro-3D-printed rock analogs. Journal of Petroleum Science and Engineering. 2021 Apr; 205: 108827.

[7] $\mathrm{Yu} \mathrm{Y,} \mathrm{Niu} \mathrm{W,} \mathrm{Yang} \mathrm{G,} \mathrm{et} \mathrm{al.} \mathrm{Mechanisms} \mathrm{for} \mathrm{the} \mathrm{accumulation} \mathrm{of} \mathrm{deep} \mathrm{gas} \mathrm{in} \mathrm{the} \mathrm{southern} \mathrm{Songliao}$ Basin, China. Journal of Petroleum Science and Engineering. 2019 Nov; 182: 106302.

[8] Yin D, Xu Q. Comparison of sandstone damage measurements based on non-destructive testing. Materials. 2020 Nov; 13(22): 5154.

[9] Sun W, Hou K, Yang Z, et al. X-ray CT three-dimensional reconstruction and discrete element analysis of the cement paste backfill pore structure under uniaxial compression. Construction and Building Materials. 2017 May; 138: 69-78.

[10] Razavifar M, Mukhametdinova A, Nikooee E, et al. Rock Porous Structure Characterization: A Critical Assessment of Various State-of-the-Art Techniques. Transport in Porous Media. 2021 Jan; 136(2): 431-456.

[11] Wei W, Yj A, Rs B, et al. Pore-throat characteristics of tight sandstone reservoirs composed of gravity flow sediments: Yingcheng Formation, Longfengshan sag, China. Journal of Petroleum Science and Engineering. 2018 Dec; 171: 646-661.

[12] Heath JE, Kuhlman KL, Broome ST, et al. Heterogeneous multiphase flow properties of volcanic rocks and implications for noble gas transport from underground nuclear explosions. Vadose Zone Journal. 2021 May; 20(3): e20123

[13] Guo L, Cheng H, Chen J, et al. Pore structure characterization of oak via X-ray computed tomography. BioResources. 2020 Mar; 15(2): 3053-3063. 